\title{
Kato decomposition of linear pencils
}

\author{
by \\ Dominique Gagnage (Lille)
}

\begin{abstract}
T. Kato [5] found an important property of semi-Fredholm pencils, now called the Kato decomposition. M. A. Kaashoek [3] introduced operators having the property $P(S: k)$ as a generalization of semi-Fredholm operators. In this work, we study this class of operators. We show that it is characterized by a Kato-type decomposition. Other properties are also proved.
\end{abstract}

1. Introduction. Throughout this paper, we shall denote by $X, Y$ two Banach spaces. Let $\mathcal{B}(X, Y)$ be the set of all bounded linear operators from $X$ to $Y$. For an operator $A$ in $\mathcal{B}(X, Y)$, we denote by $N(A)$ and $R(A)$ its kernel and range, respectively.

We will write $\mathbb{N}=\{0,1,2, \ldots\}$.

Let $T, S \in \mathcal{B}(X, Y)$. The operator $T$ is said to be semi-Fredholm if $R(T)$ is closed and $\min \{\operatorname{dim} N(T)$, codim $R(T)\}$ is finite. M. A. Kaashoek introduced the $P(S: k)$ property (see [3] and Section 2 below) as a generalization of semi-Fredholm operators.

1.1. Definition. The couple $(T, S)$ is said to have a Kato decomposition of finite type (KDF) if there exist closed subspaces $X_{1}, X_{2}$ of $X$ and $Y_{1}, Y_{2}$ of $Y$ such that:

(1) $X=X_{1} \oplus X_{2}$ and $Y=Y_{1} \oplus Y_{2}$,

(2) $\operatorname{dim} X_{1}<\infty$,

(3) $S X_{i} \subset Y_{i}$ and $T X_{i} \subset Y_{i}$, for $i=1,2$,

(4) $\left.T\right|_{X_{2}}$ has the property $P\left(\left.S\right|_{X_{2}}: 0\right)$, whose definition is recalled in Section 2,

(5) $S: X_{1} \rightarrow Y_{1}$ is bijective,

(6) $\left.S^{-1} T\right|_{X_{1}}$ is nilpotent.

In that case, we will say that $\left(X_{2}, X_{1}, Y_{2}, Y_{1}\right)$ is a KDF associated to the couple $(T, S)$.

2000 Mathematics Subject Classification: Primary 47A53. 
In [5, Theorem 4], T. Kato proved that if $T$ is semi-Fredholm and $S$ arbitrary, then $(T, S)$ has a Kato decomposition of finite type.

The aim of this paper is to characterize couples of operators having a KDF. In particular, we show that $(T, S)$ has a Kato decomposition of finite type if and only if $T$ has the property $P(S: k)$ for some integer $k$. In the particular case of $X=Y$ and $S=I$, we recover known results (see [7], [8]) about s-regular and essentially s-regular operators (also [1], [3]-[5], [6], [9]).

2. Characterization of the class $\mathcal{P}(S)$. Throughout this paper, we will consider $T, S$ in $\mathcal{B}(X, Y)$ such that $R(T)$ is closed.

T. Kato introduced sequences of subspaces of $X,\left(D_{n}(T: S)\right)_{n \geq 0}$ and $\left(N_{n}(T: S)\right)_{n \geq 0}$. They play an important role in perturbation theory (see [5]). A few years later, M. A. Kaashoek added other sequences of subspaces of $Y,\left(R_{n}(T: S)\right)_{n \geq 0}$ and $\left(M_{n}(T: S)\right)_{n \geq 0}$ (see [3]). Let us recall their definition:

$$
\left\{\begin{array}{l}
D_{0}(T: S)=X, R_{0}(T: S)=Y, \\
R_{n+1}(T: S)=T D_{n}(T: S), D_{n+1}(T: S)=S^{-1} R_{n+1}(T: S) \quad \text { for } n \geq 0, \\
N_{0}(T: S)=\{0\}, \quad M_{0}(T: S)=\{0\}, \\
N_{n+1}(T: S)=T^{-1} M_{n}(T: S), \quad M_{n+1}(T: S)=S N_{n+1}(T: S) \text { for } n \geq 0 .
\end{array}\right.
$$

If it is not ambiguous, we will write $D_{n}, N_{n}, R_{n}$ and $M_{n}$ for the corresponding subspaces. Clearly, we have $D_{n+1}=S^{-1} T D_{n}, N_{n+1}=T^{-1} S N_{n}, R_{n+1}=$ $T S^{-1} R_{n}, M_{n+1}=S T^{-1} M_{n}$. Moreover, the sequences $\left(D_{n}\right)_{n \geq 0}$ and $\left(R_{n}\right)_{n \geq 0}$ are decreasing, and the sequences $\left(N_{n}\right)_{n \geq 0}$ and $\left(M_{n}\right)_{n \geq 0}$ are increasing. Let

$$
\begin{array}{ll}
D(T: S)=\bigcap_{n=0}^{\infty} D_{n}(T: S), & N(T: S)=\bigcup_{n=0}^{\infty} N_{n}(T: S), \\
R(T: S)=\bigcap_{n=0}^{\infty} R_{n}(T: S), & M(T: S)=\bigcup_{n=0}^{\infty} M_{n}(T: S) .
\end{array}
$$

We can easily see that $T D(T: S) \subset R(T: S), S^{-1} R(T: S)=D(T: S)$ and for every complex number $\lambda \neq 0, N(T+\lambda S) \subset D(T: S)$.

We notice that in the particular case when $X=Y$ and $S=I$, we have $D_{n}=R_{n}=R\left(T^{n}\right)$ and $N_{n}=M_{n}=N\left(T^{n}\right)$.

Before giving the definition of the operators we will study in this paper, let us recall a notation. For two subspaces $M$ and $N$ of $X$, we write $M \subset_{\mathrm{e}} N$ if there exists a finite-dimensional subspace $F$ of $X$ such that $M \subset N+F$, i.e. $\operatorname{dim}[M /(M \cap N)]<\infty$. Notice that we can assume that $F$ is a subset of $M$. Now, we can introduce the notion we are interested in. Let $k$ be a positive integer. The operator $T$ is said to have the property $P(S: k)$ if

$$
\operatorname{dim}[N(T) /(D(T: S) \cap N(T))]=k
$$


and $R(T)$ is closed. We will write $T \in \mathcal{P}(S)$ if $R(T)$ is closed and $N(T) \subset_{\mathrm{e}}$ $D(T: S)$, i.e. if there exists $k$ such that $T$ has the property $P(S: k)$.

In this section, we will find other ways to characterize the property $P(S: k)$. First, let us give a condition (necessary and sufficient) for the operator $T$ to belong to $\mathcal{P}(S)$.

2.1. Proposition. (1) If $T$ has the property $P(S: k)$, then there exists a subspace $M$ of $X$ such that $S^{-1} T M=M$ and the map $\widehat{T}: X / M \rightarrow Y / T M$ defined by $\widehat{T}(x+M)=T x+T M$ is such that $n(\widehat{T}):=\operatorname{dim} N(\widehat{T})=k$.

(2) If there exists a subspace $M$ of $X$ such that $S^{-1} T M=M$ and $n(\widehat{T})=k$, with $\widehat{T}$ the map defined in $(1)$, then $T$ has the property $P\left(S: k^{\prime}\right)$ for some $k^{\prime} \leq k$.

Proof. (1) Let $M=D(T: S)$. Then, by [3, Theorem 3.1], $M=S^{-1} T M$ and $M, T M$ are closed. Let $\widehat{T}: X / M \rightarrow Y / T M$ be as in the statement. Then $N(\widehat{T})=\{x+M ; T x \in T M\}=N(T)+M$. Define $\varphi: N(T) \rightarrow N(\widehat{T})$ by $\varphi(x)=x+M$. Clearly, $\varphi$ is surjective. Further, $N(\varphi)=N(T) \cap M$, so $\varphi: N(T) /[N(T) \cap M] \rightarrow N(\widehat{T})$ is bijective. Hence $n(\widehat{T})=k$.

(2) As $S^{-1} T M=M$, we have $M \subset D(T: S)$. Let $\varphi: N(T) \rightarrow N(\widehat{T})$ be as above. Again, $\varphi: N(T) /[N(T) \cap M] \rightarrow N(\widehat{T})$ is bijective. Therefore $\operatorname{dim}[N(T) /(N(T) \cap M)]=\operatorname{dim} N(\widehat{T})=k$. Since $M \subset D(T: S)$, it follows that $\operatorname{dim}[N(T) /(N(T) \cap D(T: S))] \leq k$. Thus $T$ has the property $P\left(S: k^{\prime}\right)$ for some $k^{\prime} \leq k$, as $R(T)$ is supposed to be closed.

Remark. In both parts of Proposition 2.1, $M$ and $T M$ are closed and $\widehat{T}$ has closed range.

Proof. As $N(\widehat{T})=N(T)+M$ is finite-dimensional, it is closed. Moreover, $T$ has closed range. So, by [5, Lemma 331], $T N(\widehat{T})=T M$ is closed. As $M=S^{-1} T M$, the subspace $M$ is also closed. Let $\Pi: Y \rightarrow Y / T M$ be the canonical projection. Since $N(\Pi)=T M \subset R(T)$ and $R(T)$ is closed, $R(\widehat{T})=\Pi R(T)$ is closed, by [5, Lemma 331].

2.2. Corollary. $T \in \mathcal{P}(S)$ if and only if there exists a closed subspace $M$ of $X$ such that $S^{-1} T M=M$ and the map $\widehat{T}: X / M \rightarrow Y / T M$ defined by $\widehat{T}(x+M)=T x+T M$ is upper semi-Fredholm.

The main result of this work is the following theorem which allows us to characterize operators having the property $\mathcal{P}(S)$ in terms of a Kato decomposition of finite type.

2.3. Theorem. Let $T, S \in \mathcal{B}(X, Y)$ be such that $R(T)$ is closed. The following are equivalent:

(1) $T \in \mathcal{P}(S)$.

(2) $N(T: S) \subset_{\mathrm{e}} S^{-1} R(T)$. 
(3) $N(T: S) \subset_{\mathrm{e}} D(T: S)$.

(4) The couple $(T, S)$ has a Kato decomposition of finite type.

The following lemmas will allow us to prove Theorem 2.3.

2.4. Lemma. Let $U, V, W$ be subspaces of $X$ and $M, N$ be subspaces of $Y$.

(1) $T[U \cap V] \subset T U \cap T V$, with equality if $N(T) \subset U$.

(2) $S^{-1} M+S^{-1} N \subset S^{-1}[M+N]$, with equality if $N \subset R(S)$.

(3) Let $A \in \mathcal{B}(X, Y)$ be a closed range operator. If $U+N(A)$ is closed, then $A U$ is closed.

(4) If $U \subset W$, then $[U+V] \cap W=U+[V \cap W]$.

Proof. (1) The inclusion is clear. Assume that $N(T) \subset U$ and let $y \in$ $T U \cap T V$. There exist $u \in U$ and $v \in V$ such that $y=T u=T v$. Then $v-u \in N(T) \subset U$, and so $v \in U \cap V$. Thus $y \in T[U \cap V]$.

(2) Let $x \in S^{-1} M+S^{-1} N$. Then there exist $m \in S^{-1} M$ and $n \in S^{-1} N$ such that $x=m+n$. Thus $S x \in M+N$, and so $x \in S^{-1}[M+N]$.

Now, assume that $N \subset R(S)$ and let $x \in S^{-1}[M+N]$. Then there exist $a \in M$ and $b \in N \subset R(S)$ such that $S x=a+b$. Moreover, there exists $c \in X$ such that $b=S c$, and so $c \in S^{-1} N$. Thus $S(x-c)=a \in M$, and $x-c \in S^{-1} M$. Hence $x \in S^{-1} N+S^{-1} M$.

(3) [5, Lemma 331].

(4) [2, Lemma 2.1].

2.5. Lemma. The following are equivalent:

(1) For all $n \in \mathbb{N}, N_{n}(T: S) \subset S^{-1} R(T)$, i.e. $N(T: S) \subset S^{-1} R(T)$.

(2) $N(T) \subset D(T: S)$, i.e. $T$ has the property $P(S: 0)$.

(3) For all $(n, k) \in \mathbb{N}^{2}, N_{n}(T: S) \subset D_{k}(T: S)$, i.e. $N(T: S) \subset D(T: S)$.

Proof. $(3) \Rightarrow(1):$ As $D(T: S) \subset D_{1}(T: S)=S^{-1} R(T)$, we obtain $N(T: S) \subset S^{-1} R(T)$.

$(1) \Rightarrow(3)$ : We argue by induction on $k \in \mathbb{N}$. As the cases $k=0,1$ are true, assume that the property holds for some $k \geq 1$. Let $n \in \mathbb{N}$. We have $N_{n+1}(T: S) \subset D_{k}(T: S)$. As $N_{n+1}(T: S)=T^{-1} S N_{n}(T: S)$, it follows that

$$
S N_{n}(T: S) \cap R(T) \subset T D_{k}(T: S) .
$$

Then $\left[N_{n}(T: S)+N(S)\right] \cap S^{-1} R(T) \subset S^{-1} T D_{k}(T: S)=D_{k+1}(T: S)$. By Lemma 2.4, as $N(S) \subset S^{-1} R(T)$, we have

$$
N_{n}(T: S) \cap S^{-1} R(T)+N(S) \subset D_{k+1}(T: S) .
$$

Since $N_{n}(T: S) \subset S^{-1} R(T)$, we obtain the result.

$(3) \Rightarrow(2)$ : As $N(T)=N_{1}(T: S) \subset N(T: S)$, we obtain $N(T) \subset D(T: S)$.

$(2) \Rightarrow(3)$ : We reason by induction on $n \in \mathbb{N}$. As the cases $n=0,1$ are true, assume that the property holds for some $n \geq 1$. Let $k \in \mathbb{N}$. We have 
$N_{n}(T: S) \subset D_{k+1}(T: S)$. As $D_{k+1}(T: S)=S^{-1} T D_{k}(T: S)$, it follows that

$$
S N_{n}(T: S) \subset T D_{k}(T: S) \cap R(S) \subset T D_{k}(T: S) .
$$

Then $N_{n+1}(T: S)=T^{-1} S N_{n}(T: S) \subset D_{k}(T: S)+N(T)=D_{k}(T: S)$, as $N(T) \subset D_{k}(T: S)$.

2.6. Lemma. Consider the following properties:

(a) $N(T) \subset_{\mathrm{e}} D(T: S)$, i.e. $T \in \mathcal{P}(S)$.

(b) $N(T: S) \subset_{\mathrm{e}} S^{-1} R(T)$.

(c) For all $(n, p) \in \mathbb{N}^{2}, N_{n}(T: S) \subset_{\mathrm{e}} D_{p}(T: S)$.

We have the following implications: $(\mathrm{a}) \Rightarrow(\mathrm{c})$ and $(\mathrm{b}) \Rightarrow(\mathrm{c})$.

Proof. (a) $\Rightarrow$ (c): Assuming (a), we show (c) by induction on $n$. Since $D(T: S) \subset D_{p}(T: S)$ for every $p \in \mathbb{N}$, the cases $n=0,1$ are clear. Let $n \geq 1$ and assume that for all $m \leq n$ and all $p \in \mathbb{N}$, there exists a finite-dimensional subspace $F_{m, p} \subset N_{m}(T: S)$ such that $N_{m}(T: S) \subset D_{p}(T: S)+F_{m, p}$.

Let $p \in \mathbb{N}$. Then

$$
\begin{aligned}
N_{n+1}(T: S) & =T^{-1} S N_{n}(T: S) \subset T^{-1} S\left[D_{p+1}(T: S)+F_{n, p+1}\right] \\
& =T^{-1}\left[S D_{p+1}(T: S)+S F_{n, p+1}\right] \\
& =T^{-1}\left[R_{p+1}(T: S) \cap R(S)+S F_{n, p+1}\right] \\
& \subset T^{-1}\left[R_{p+1}(T: S)+S F_{n, p+1}\right] \\
& =T^{-1}\left[T D_{p}(T: S)+S F_{n, p+1}\right] \\
& =D_{p}(T: S)+N(T)+T^{-1} S F_{n, p+1} \quad \text { by Lemma } 2.4 .
\end{aligned}
$$

As $S F_{n, p+1} \cap R(T)$ is finite-dimensional, we can find $F_{n+1, p}^{\prime} \subset X$ such that

$$
\left\{\begin{array}{l}
T F_{n+1, p}^{\prime}=S F_{n, p+1} \cap R(T), \\
\operatorname{dim} F_{n+1, p}^{\prime}=\operatorname{dim}\left[S F_{n, p+1} \cap R(T)\right] .
\end{array}\right.
$$

Thus $N_{n+1}(T: S) \subset D_{p}(T: S)+F_{n+1, p}^{\prime}+N(T)$. Further $N(T) \subset F_{1, p}+$ $D_{p}(T: S)$. Defining $F_{n+1, p}=F_{1, p}+F_{n+1, p}^{\prime}$, a finite-dimensional subspace, we have $N_{n+1}(T: S) \subset D_{p}(T: S)+F_{n+1, p}$.

(b) $\Rightarrow$ (c): Assuming (b), we show (c) by induction on $p$. As $N_{n}(T: S) \subset$ $N(T: S)$ for all $n \in \mathbb{N}$, the cases $p=0,1$ are clear. Let $p \geq 1$ and assume that for all $q \leq p$ and all $n \in \mathbb{N}$, there exists a finite-dimensional subspace $F_{n, q} \subset N_{n}(T: S)$ such that $N_{n}(T: S) \subset D_{q}(T: S)+F_{n, q}$.

Let $n \in \mathbb{N}$. We have

$$
\begin{aligned}
T N_{n+1}(T: S) & \subset T\left[D_{p}(T: S)+F_{n+1, p}\right]=T D_{p}(T: S)+T F_{n+1, p} \\
& =R_{p+1}(T: S)+T F_{n+1, p} .
\end{aligned}
$$

As $F_{n+1, p} \subset N_{n+1}(T: S)$, we obtain

$$
T F_{n+1, p} \subset T N_{n+1}(T: S) \subset M_{n}(T: S)=S N_{n}(T: S) \subset R(S) .
$$


Thus

$$
\begin{aligned}
S^{-1} T N_{n+1}(T: S) & \subset S^{-1} R_{p+1}(T: S)+S^{-1} T F_{n+1, p} \quad \text { by Lemma } 2.4 \\
& =D_{p+1}(T: S)+S^{-1} T F_{n+1, p} .
\end{aligned}
$$

Since $T N_{n+1}(T: S)=S N_{n}(T: S) \cap R(T)$, we have

$$
\begin{aligned}
S^{-1} T N_{n+1}(T: S) & =\left[N_{n}(T: S)+N(S)\right] \cap S^{-1} R(T) \\
& =\left[N_{n}(T: S) \cap S^{-1} R(T)\right]+N(S) \quad \text { by Lemma } 2.4 \\
& \supset N_{n}(T: S) \cap D_{1}(T: S) .
\end{aligned}
$$

Moreover, $N_{n}(T: S) \subset D_{1}(T: S)+F_{n, 1}$ with $F_{n, 1} \subset N_{n}(T: S)$. So

$$
\begin{aligned}
N_{n}(T: S) & \subset D_{1}(T: S) \cap N_{n}(T: S)+F_{n, 1} \subset S^{-1} T N_{n+1}(T: S)+F_{n, 1} \\
& \subset D_{p+1}(T: S)+S^{-1} T F_{n+1, p}+F_{n, 1} .
\end{aligned}
$$

Notice that $T F_{n+1, p} \cap R(S)$ is finite-dimensional. Let $F_{n+1, p}^{\prime}$ be such that

$$
\left\{\begin{array}{l}
S F_{n+1, p}^{\prime}=T F_{n+1, p} \cap R(S), \\
\operatorname{dim} F_{n+1, p}^{\prime}=\operatorname{dim}\left(T F_{n+1, p} \cap R(S)\right) .
\end{array}\right.
$$

Thus $N_{n}(T: S) \subset D_{p+1}(T: S)+F_{n, 1}+F_{n+1, p}^{\prime}$, as $N(S) \subset D_{p+1}(T: S)$. Setting $F_{n, p+1}=F_{n, 1}+F_{n+1, p}^{\prime}$, a finite-dimensional subspace, we obtain

$$
N_{n}(T: S) \subset D_{p+1}(T: S)+F_{n, p+1} .
$$

2.7. Lemma. For all $(m, d) \in \mathbb{N}^{2}$,

$$
\left(S^{-1} T\right)^{d} N_{m+d}(T: S)=N_{m}(T: S) \cap D_{d}(T: S)+N_{d}(S: T) .
$$

Notice that in the last term, the roles of $T$ and $S$ are reversed. This will happen at some other places as well.

Proof. We prove by induction on $d \in \mathbb{N}$ that the stated equality holds for all integers $m$. As the case $d=0$ is clear, assume that the equality holds for some $d \geq 0$ and let $m \in \mathbb{N}$. Thanks to Lemma 2.4, we have

$$
\begin{aligned}
\left(S^{-1} T\right)^{d+1} & N_{m+d+1}(T: S)=\left(S^{-1} T\right)\left[\left(S^{-1} T\right)^{d} N_{(m+1)+d}(T: S)\right] \\
& =\left(S^{-1} T\right)\left[N_{m+1}(T: S) \cap D_{d}(T: S)+N_{d}(S: T)\right] \\
& =S^{-1}\left[M_{m}(T: S) \cap R_{d+1}(T: S)+M_{d}(S: T)\right] \\
& =S^{-1}\left[S N_{m}(T: S) \cap R_{d+1}(T: S)+M_{d}(S: T)\right] \\
& =\left[N_{m}(T: S)+N(S)\right] \cap S^{-1} R_{d+1}(T: S)+S^{-1} M_{d}(S: T) \\
& =N_{m}(T: S) \cap D_{d+1}(T: S)+N(S)+N_{d+1}(S: T) \\
& =N_{m}(T: S) \cap D_{d+1}(T: S)+N_{d+1}(S: T) .
\end{aligned}
$$

2.8. Lemma. Assume that $N_{k}(T: S) \subset D_{1}(T: S)$ for all $k \in\{0, \ldots, p\}$. Then $N_{p-j}(T: S) \subset D_{j+1}(T: S)$ for each $j \in\{0, \ldots, p\}$. 
Proof. We prove this by induction on $j$. The result is clear for $j=0$. Assume that the property is true for some $j \in\{0, \ldots, p-1\}$. As

$$
T^{-1} S N_{p-(j+1)}(T: S)=N_{p-j}(T: S) \subset D_{j+1}(T: S),
$$

we have $S N_{p-j-1}(T: S) \cap R(T) \subset T D_{j+1}(T: S)$. So

$$
\left[N_{p-j-1}(T: S)+N(S)\right] \cap S^{-1} R(T) \subset D_{j+2}(T: S) .
$$

Since $N_{p-j-1}(T: S)$ and $N(S)$ are included in $D_{1}(T: S)=S^{-1} R(T)$, we obtain

$$
N_{p-(j+1)}(T: S) \subset N_{p-j-1}(T: S)+N(S) \subset D_{j+2}(T: S) .
$$

2.9. Lemma. Assume that $N(T) \subset_{\mathrm{e}} D_{k}(T: S)$ for all $k \in \mathbb{N}$. Then, for each $j \in \mathbb{N}, D_{j}(T: S)$ and $R_{j}(T: S)$ are closed.

Proof. As $D_{0}(T: S)$ and $R_{0}(T: S)$ are closed, assume that the property is true for some $j \in \mathbb{N}$. As $\operatorname{dim}\left[N(T) /\left(N(T) \cap D_{j}(T: S)\right)\right]<\infty$, there exists a finite-dimensional subspace $N$ of $N(T)$ such that $N \oplus D_{j}(T: S)=N(T)+$ $D_{j}(T: S)$ and $N \cap D_{j}(T: S)=\{0\}$.

Let $M=D_{j}(T: S) \oplus N=D_{j}(T: S)+N(T)$. The subspace $M$ is closed and $T M=R_{j+1}(T: S)$. Since $N(T) \subset M$ and $R(T)$ and $M$ are closed, $R_{j+1}(T: S)$ is closed, by Lemma 2.4 . As $S$ is continuous, $S^{-1} R_{j+1}(T: S)=$ $D_{j+1}(T: S)$ is also closed.

2.10. Lemma. Assume that there exists $n \in \mathbb{N} \backslash\{0\}$ such that $N_{n}(T: S)$ $\nsubseteq S^{-1} R(T)$ and $N_{n-1}(T: S) \subset S^{-1} R(T)$. Let $y \in N_{n}(T: S) \backslash S^{-1} R(T)$. Then $y,\left(S^{-1} T\right) y, \ldots,\left(S^{-1} T\right)^{n-1} y$ are independent modulo $D_{n}(T: S)$.

Proof. First, we prove that

$$
\left(S^{-1} T\right)^{j} y \subset N_{n-j}(T: S)+N_{j}(S: T) \text { for } j=0, \ldots, n .
$$

This is clear for $j=0$. Assume that it is true for some $j \in\{0, \ldots, n-1\}$. Then $\left(S^{-1} T\right)^{j} y \subset N_{n-j}(T: S)+N_{j}(S: T)=T^{-1} S N_{n-j-1}(T: S)+$ $N_{j}(S: T)$. So

$$
\begin{aligned}
T\left(S^{-1} T\right)^{j} y & \subset S N_{n-j-1}(T: S) \cap R(T)+T N_{j}(S: T) \\
& \subset S N_{n-j-1}(T: S)+T N_{j}(S: T) .
\end{aligned}
$$

Thus, by Lemma 2.4, we have

$$
\begin{aligned}
\left(S^{-1} T\right)^{j+1} y & \subset N_{n-j-1}(T: S)+N(S)+N_{j+1}(S: T) \\
& =N_{n-j-1}(T: S)+N_{j+1}(S: T) .
\end{aligned}
$$

We now prove that

$$
\left(S^{-1} T\right)^{j} y \cap D_{j+1}(T: S)=\emptyset \quad \text { for } j=0, \ldots, n-1 .
$$

The case $j=0$ is clear. Assume that the property is true for some $j \in$ $\{0, \ldots, n-2\}$ and that there exists $x$ in $\left(S^{-1} T\right)^{j+1} y \cap D_{j+2}(T: S)$. Then 
$S x=T z$ for some $z$ in $\left(S^{-1} T\right)^{j} y$. We have $z \in T^{-1} S x \subset T^{-1} S D_{j+2}(T: S)$. As $D_{j+2}(T: S)=S^{-1} T D_{j+1}(T: S)$, it follows that

$$
S D_{j+2}(T: S)=T D_{j+1}(T: S) \cap R(S) \subset T D_{j+1}(T: S),
$$

and $T^{-1} S D_{j+2}(T: S) \subset D_{j+1}(T: S)+N(T)$. By Lemma 2.8 , as $N_{k}(T: S)$ $\subset D_{1}(T: S)$ for all $k \in\{0, \ldots, j+1\}(j+1 \leq n-1)$, we have $N(T) \subset$ $D_{j+1}(T: S)$. So $T^{-1} S D_{j+2}(T: S) \subset D_{j+1}(T: S)$. Thus $z \in D_{j+1}(T: S) \cap$ $\left(S^{-1} T\right)^{j} y$, a contradiction.

Now, we can prove that $y,\left(S^{-1} T\right) y, \ldots,\left(S^{-1} T\right)^{n-1} y$ are independent modulo $D_{n}(T: S)$. Let $z_{j} \in\left(S^{-1} T\right)^{j} y$ and $\alpha_{j} \in \mathbb{C}$ for $j=0, \ldots, n-1$. Assume that $\alpha_{0} z_{0}+\ldots+\alpha_{n-1} z_{n-1}$ belongs to $D_{n}(T: S)$. Then, by Lemma 2.4 , applying $\left(S^{-1} T\right)^{n-1}$, we obtain

$$
\begin{aligned}
\alpha_{0}\left(S^{-1} T\right)^{n-1} z_{0}+\ldots+\alpha_{n-1}\left(S^{-1} T\right)^{n-1} z_{n-1} & \subset\left(S^{-1} T\right)^{n-1} D_{n}(T: S) \\
& =D_{2 n-1}(T: S) .
\end{aligned}
$$

For every $k \in \mathbb{N}, N(S) \subset D_{k}(T: S)$, so $N_{j+1}(S: T)=\left(S^{-1} T\right)^{j} N(S) \subset$ $D_{k+j}(T: S)$ for all $(k, j) \in \mathbb{N}^{2}$. Since the sequence $\left(D_{k}(T: S)\right)_{k \geq 0}$ is decreasing, we have

$$
N_{j}(S: T) \subset D_{k}(T: S) \quad \text { for each couple }(k, j) \in \mathbb{N}^{2} .
$$

For $j \in\{0, \ldots, n-1\}$, we have

$$
\begin{aligned}
z_{j} & \in\left(S^{-1} T\right)^{j} y \subset N_{n-j}(T: S)+N_{j}(S: T) \quad \text { by }(1) \\
& \subset D_{j}(T: S)+N_{j}(S: T) \quad \text { by Lemma } 2.8 \\
& \subset D_{j}(T: S) \quad \text { by }(3) .
\end{aligned}
$$

So $\left(S^{-1} T\right)^{n-1} z_{j} \subset\left(S^{-1} T\right)^{n-1} D_{j}(T: S)=D_{n+j-1}(T: S)$ for $j=1, \ldots, n-1$. As the sequence $\left(D_{k}(T: S)\right)_{k \geq 0}$ is decreasing, we obtain

$$
\alpha_{1}\left(S^{-1} T\right)^{n-1} z_{1}+\ldots+\alpha_{n-1}\left(S^{-1} T\right)^{n-1} z_{n-1} \subset D_{n}(T: S) .
$$

Hence $\alpha_{0}\left(S^{-1} T\right)^{n-1} z_{0}=\alpha_{0}\left(S^{-1} T\right)^{n-1} y \subset D_{n}(T: S)$.

If $\alpha_{0} \neq 0$, we have found an element belonging to $D_{n}(T: S)$ and to $\left(S^{-1} T\right)^{n-1} y$, which contradicts (2).

So $\alpha_{0}=0$. Thus $\alpha_{1} z_{1}+\ldots+\alpha_{n-1} z_{n-1} \in D_{n}(T: S)$. Applying $\left(S^{-1} T\right)^{n-2}$, we show that $\alpha_{1}=0$. Step by step, we conclude that $\alpha_{i}=0$ for $i=$ $0, \ldots, n-1$.

Proof of Theorem 2.3. The implications $(3) \Rightarrow(1)$ and $(3) \Rightarrow(2)$ are clear since $N(T)=N_{1}(T: S) \subset N(T: S)$ and $D(T: S) \subset D_{1}(T: S)=S^{-1} R(T)$.

$(4) \Rightarrow(3)$ : Let $\left(X_{2}, X_{1}, Y_{2}, Y_{1}\right)$ be a KDF associated to the couple $(T, S)$. We have

$N(T: S)=N\left(T_{2}: S_{2}\right) \oplus N\left(T_{1}: S_{1}\right) \subset N\left(T_{2}: S_{2}\right)+X_{1} \subset D\left(T_{2}: S_{2}\right)+X_{1}$, by Lemma 2.5, since $\left.T\right|_{X_{2}}$ has the property $P\left(\left.S\right|_{X_{2}}: 0\right)$. As $\left.S^{-1} T\right|_{X_{1}}$ is nilpotent of index $d, D_{n}(T: S)=D_{n}\left(T_{1}: S_{1}\right) \oplus D_{n}\left(T_{2}: S_{2}\right)=D_{n}\left(T_{2}: S_{2}\right)$ 
for all $n \geq d$. Therefore $D(T: S)=D\left(T_{2}: S_{2}\right)$. Moreover $X_{1}$ is finitedimensional, so we have $N(T: S) \subset_{\mathrm{e}} D(T: S)$.

We now prove that (1) or (2) implies (4). In these two cases, (c) of Lemma 2.6 is satisfied. Thus, for all positive integers $n$, we have $N_{n}(T: S) \subset_{\mathrm{e}}$ $D_{1}(T: S)=S^{-1} R(T)$.

If $N_{n}(T: S) \subset S^{-1} R(T)$ for all $n \geq 0$, then by Lemma 2.5, $T$ has the property $P(S: 0)$, and $(X,\{0\}, Y,\{0\})$ is a Kato decomposition of finite type associated to $(T, S)$.

Assume that there exists $n \geq 1$ such that $N_{n}(T: S) \nsubseteq S^{-1} R(T)$ and $N_{n-1}(T: S) \subset S^{-1} R(T)$. Let $y \in N_{n}(T: S) \backslash S^{-1} R(T)$. By Lemma 2.10, $y,\left(S^{-1} T\right) y, \ldots,\left(S^{-1} T\right)^{n-1} y$ are independent modulo $D_{n}(T: S)$. As $y \in$ $N_{n}(T: S)$, there exist $x_{0}, \ldots, x_{n}$ such that $S x_{i+1}=T x_{i}$ for $i=0, \ldots, n-1$, $x_{0}=y$ and $x_{n}=0$. Let $z_{i}=S x_{i}$ for $i=0, \ldots, n-1$. We prove that $z_{0}, \ldots, z_{n-1}$ are independent. If $\alpha_{0} z_{0}+\ldots+\alpha_{n-1} z_{n-1}=0$, then $\alpha_{0} x_{0}+$ $\ldots+\alpha_{n-1} x_{n-1} \in N(S) \subset D_{n}(T: S)$. As $y,\left(S^{-1} T\right) y, \ldots,\left(S^{-1} T\right)^{n-1} y$ are independent modulo $D_{n}(T: S), \alpha_{i}=0$ for $i=0, \ldots, n-1$. Notice that $x_{0}, \ldots, x_{n-1}$ are also independent.

Step 1. We will define two projections $P$ and $Q$ which will allow us to find a Kato decomposition. To do so, we first define a functional $f \in X^{*}$ which will be useful in the construction of $P$ and $Q$.

As (c) of Lemma 2.6 holds, by Lemma $2.9, D_{j}(T: S)$ is closed for all positive integers $j$. In particular, $D_{n}(T: S)$ is closed. So, by the HahnBanach Theorem, there exists $f \in D_{n}(T: S)^{\perp}$ such that $f\left(x_{n-1}\right)=1$ and $f\left(x_{j}\right)=0$ for $j=0, \ldots, n-2$.

Let us prove that

$$
\left.f\right|_{\left(S^{-1} T\right)^{j} y}=\delta_{j, n-1} \quad \text { for } j=0, \ldots, n-1 .
$$

This is clear for $j=0$. Let $j \in\{1, \ldots, n-1\}$ and $a_{j} \in\left(S^{-1} T\right)^{j} y$. There exist $u_{0}, \ldots, u_{j}, v_{0}, \ldots, v_{j}$ such that $u_{0}=x_{j}, v_{0}=a_{j}, u_{j}=v_{j}=y, S u_{i}=T u_{i+1}$ and $S v_{i}=T v_{i+1}$. Then $u_{j-1}-v_{j-1} \in N(S)$. Step by step, we obtain $u_{0}-v_{0} \in N_{j}(S: T) \subset D_{n}(T: S)$. As $f \in D_{n}(T: S)^{\perp}$, we find that $f\left(u_{0}\right)=f\left(v_{0}\right)$, i.e. $f\left(x_{j}\right)=f\left(a_{j}\right)$.

Let us show some properties of $f$. Set $K_{i, j}=\left(S^{-1} T\right)^{i}\left(S^{-1} T\right)^{n-j-1} y$ for $0 \leq j \leq n-1$.

We prove that

$$
\left.f\right|_{K_{i, j}}=\delta_{i j} \quad \text { for } 0 \leq i, j \leq n-1 .
$$

If $i=j$, then $K_{i, i}=\left(S^{-1} T\right)^{n-1} y$, so $\left.f\right|_{K_{i, i}}=1$.

If $i>j$, then $K_{i, j}=\left(S^{-1} T\right)^{n+(i-j-1)} y \subset\left(S^{-1} T\right)^{n+(i-j-1)} N_{n}(T: S)$. Moreover, by Lemma 2.7, for all positive integers $k$, we have 


$$
\begin{aligned}
\left(S^{-1} T\right)^{p} & N_{k}(T: S) \\
& = \begin{cases}N_{k-p}(T: S) \cap D_{p}(T: S)+N_{p}(S: T) & \text { if } p \in\{0, \ldots, k\}, \\
N_{p}(S: T) & \text { if } p \geq k .\end{cases}
\end{aligned}
$$

Thus $K_{i, j} \subset N_{n+i-j-1}(S: T) \subset D_{n}(T: S)$, so $K_{i, j} \subset D_{n}(T: S)$. Hence $\left.f\right|_{K_{i, j}}=0$, as $f \in D_{n}(T: S)^{\perp}$.

If $i<j$, then $0 \leq(n-1)-(j-i) \leq n-2$, hence also $\left.f\right|_{K_{i, j}}=0$.

We now prove that

$\forall x \in X, \forall j \in\{0, \ldots, n-1\}, \quad f$ is constant on $H_{j}(x)=\left(s^{-1} T\right)^{j} x$.

Let $x \in X, j \in\{0, \ldots, n-1\}$ and consider $z_{1}, z_{2}$ in $H_{j}(x)$. For $i=1,2$, there exist $h_{i}^{0}, \ldots, h_{i}^{j}$ such that $h_{i}^{0}=z_{i}, h_{i}^{j}=x$ and $S h_{i}^{p-1}=T h_{i}^{p}$ for $1 \leq p \leq j$. We can easily show that $h_{1}^{j-p}-h_{2}^{j-p} \in N_{p}(S: T)$ for $p \in\{0, \ldots, j\}$. So $z_{1}-z_{2} \in N_{j}(S: T) \subset D_{n}(T: S)$, hence $f\left(z_{1}\right)=f\left(z_{2}\right)$. This will allow us to define the projection $P$.

Next, we show that

$$
\forall z \in Y, \forall j \in\{0, \ldots, n-1\}, \quad f \text { is constant on } H_{j}\left(S^{-1} z\right),
$$

which will allow us to construct $Q$. Let $z \in Y, j \in\{0, \ldots, n-1\}$ and $z_{1}, z_{2}$ be in $H_{j}\left(S^{-1} z\right)$. For $i=1,2$, there exist $h_{i}^{0}, \ldots, h_{i}^{j}$ such that $h_{i}^{0}=z_{i}, S h_{i}^{j}=z$ and $S h_{i}^{p-1}=T h_{i}^{p}$ for $1 \leq p \leq j$. We can easily show that $h_{1}^{j-p}-h_{2}^{j-p} \in$ $N_{p+1}(S: T)$ for $p \in\{0, \ldots, j\}$. So $z_{1}-z_{2} \in N_{j+1}(S: T) \subset D_{n}(T: S)$, and hence $f\left(z_{1}\right)=f\left(z_{2}\right)$.

Now we can define the two projections $P$ and $Q$. For $(x, z) \in X \times Y$, set

$$
P(x)=\sum_{j=0}^{n-1} f\left(H_{j}(x)\right) x_{n-j-1}, \quad Q(z)=\sum_{j=0}^{n-1} f\left(H_{j}\left(S^{-1} z\right)\right) z_{n-j-1} .
$$

We prove that

$$
P^{2}=P \quad \text { and } \quad Q^{2}=Q
$$

For $x \in X$,

$$
P^{2}(x)=\sum_{j=0}^{n-1} f\left(H_{j}(P(x))\right) x_{n-j-1} .
$$

We show that $f\left(H_{j}(P(x))\right)=f\left(H_{j}(x)\right)$. Indeed,

$$
\begin{aligned}
H_{j}(P(x)) & =\left(S^{-1} T\right)^{j}\left[\sum_{i=0}^{n-1} f\left(H_{i}(x)\right) x_{n-i-1}\right] \\
& \supset \sum_{i=0}^{n-1} f\left(H_{i}(x)\right) H_{j}\left(x_{n-i-1}\right) \quad \text { by Lemma } 2.4 .
\end{aligned}
$$


Let $w \in \sum_{i=0}^{n-1} f\left(H_{i}(x)\right) H_{j}\left(x_{n-i-1}\right)$. As $f$ is constant on each $H_{j}\left(x_{n-i-1}\right)$ $(i \in\{0, \ldots, n-1\})$, we have

$$
f(w)=\sum_{i=0}^{n-1} f\left(H_{i}(x)\right) f\left(H_{j}\left(x_{n-i-1}\right)\right) .
$$

As $x_{n-i-1} \in\left(S^{-1} T\right)^{n-i-1} y$ and $\left.f\right|_{K_{i, j}}=\delta_{i j}$, we have $f\left(H_{j}\left(x_{n-i-1}\right)\right)=$ $\delta_{i, j}$. Thus $f(w)=f\left(H_{j}(x)\right)$. As $f$ is constant on $H_{j}(P(x))$, it follows that $f\left(H_{j}(P(x))\right)=f(w)=f\left(H_{j}(x)\right)$. Therefore $P^{2}=P$.

For $z \in Y$,

$$
Q^{2}(z)=\sum_{j=0}^{n-1} f\left(H_{j}\left(S^{-1} Q(z)\right)\right) z_{n-j-1}
$$

We show that $f\left(H_{j}\left(S^{-1} Q(z)\right)\right)=f\left(H_{j}\left(S^{-1} z\right)\right)$. We have

$$
H_{j}\left(S^{-1} Q(z)\right) \supset \sum_{i=0}^{n-1} f\left(H_{i}\left(S^{-1} z\right)\right) H_{j}\left(S^{-1} z_{n-i-1}\right) \quad \text { by Lemma } 2.4 .
$$

Notice that $x_{n-i-1} \in S^{-1} z_{n-i-1}$. So $f\left[H_{j}\left(S^{-1} z_{n-i-1}\right)\right]=f\left[H_{j}\left(x_{n-i-1}\right)\right]$. Let $w \in \sum_{i=0}^{n-1} f\left(H_{i}\left(S^{-1} z\right)\right) H_{j}\left(x_{n-i-1}\right)$. As before, we find that $f(w)=$ $f\left(H_{j}\left(S^{-1} z\right)\right)$. Hence $f\left(H_{j}\left(S^{-1} Q(z)\right)\right)=f\left(H_{j}\left(S^{-1} z\right)\right)$.

Step 2. We study different properties of the two projections. Set

$$
X_{1}=R(P), \quad X_{2}=N(P), \quad Y_{1}=R(Q), \quad Y_{2}=N(Q) .
$$

We have $\operatorname{dim} X_{1}=\operatorname{dim} Y_{1}<\infty$, the equality following from the fact that $P\left(x_{k}\right)=x_{k}$ and $Q\left(z_{k}\right)=z_{k}$ for all $k \in\{0, \ldots, n-1\}$.

We want to prove that

$$
Q S=S P \quad \text { and } \quad Q T=T P .
$$

Let $x \in X$. As $x \in S^{-1} S x$ and $f$ is constant on each $H_{j}\left(S^{-1} z\right)$, it follows that $f\left(H_{j} S^{-1} S x\right)=f\left(H_{j} x\right)$. Thus, we have

$$
\begin{aligned}
Q S(x) & =\sum_{j=0}^{n-1} f\left(H_{j}(x)\right) z_{n-j-1}=\sum_{j=0}^{n-1} f\left(H_{j}(x)\right) S x_{n-j-1}=S P(x), \\
Q T(x) & =\sum_{j=0}^{n-1} f\left(H_{j} S^{-1} T x\right) z_{n-j-1}=\sum_{j=0}^{n-1} f\left(H_{j+1}(x)\right) z_{n-j-1} \\
& =\sum_{j=1}^{n-1} f\left(H_{j}(x)\right) z_{n-j} \quad \text { as } f\left(\left(S^{-1} T\right)^{n} x\right)=0 \\
& =T P(x) \quad \text { as } x_{n-1} \in N(T) .
\end{aligned}
$$

Hence $T X_{i} \subset Y_{i}$ and $S X_{i} \subset Y_{i}$ for $i=1,2$. Denote by $S_{i}$ and $T_{i}$ the restrictions of $S$ and $T$ to $X_{i}$, considered as operators from $X_{i}$ to $Y_{i}$. 
Let us prove that

$$
N(T) \cap X_{1}=\mathbb{C} x_{n-1} .
$$

Let $x \in N(T) \cap X_{1}$. As $x=P(x)$, we have

$$
0=T x=\sum_{j=1}^{n-1} f\left(H_{j}(x)\right) T x_{n-j-1}=\sum_{j=1}^{n-1} f\left(H_{j}(x)\right) S x_{n-j}=\sum_{j=1}^{n-1} f\left(H_{j}(x)\right) z_{n-j} .
$$

As $z_{0}, \ldots, z_{n-1}$ are independent, $f\left(H_{j}(x)\right)=0$ for each $j \in\{1, \ldots, n-1\}$. Thus $x=f(x) x_{n-1}$, and so $N(T) \cap X_{1} \subset \mathbb{C} x_{n-1}$. Conversely, let $x=\lambda x_{n-1}$. Then $f(x)=\lambda f\left(x_{n-1}\right)=\lambda$, so $x=f(x) x_{n-1}$. Moreover, $f(x) x_{n-1}=P(x)$, so $x \in R(P)=X_{1}$. Further, $T x=f(x) T x_{n-1}=0$.

We now prove that

$$
N(S) \cap X_{1}=\{0\} .
$$

Let $x \in N(S) \cap X_{1}$. Then $x=P(x)=\sum_{j=0}^{n-1} f\left(H_{j}(x)\right) x_{n-j-1}$. We have

$$
0=S x=S P(x)=\sum_{j=0}^{n-1} f\left(H_{j}(x)\right) S x_{n-j-1}=\sum_{j=0}^{n-1} f\left(H_{j}(x)\right) z_{n-j-1} .
$$

So $f\left(H_{j}(x)\right)=0$ for $j \in\{0, \ldots, n-1\}$, since $z_{0}, \ldots, z_{n-1}$ are independent. Hence $x=0$.

Thus $S_{1}: X_{1} \rightarrow Y_{1}$ is injective. Moreover, $\operatorname{dim} X_{1}=\operatorname{dim} Y_{1}<\infty$. So $S_{1}: X_{1} \rightarrow Y_{1}$ is bijective. Thus, we can consider $S^{-1} T$ from $X_{1}$ to $X_{1}$.

We now prove that

$$
S^{-1} T: X_{1} \rightarrow X_{1} \text { is nilpotent. }
$$

Let $x \in X_{1}$. We have

$$
T P(x)=\sum_{j=0}^{n-1} f\left(H_{j}(x)\right) T x_{n-j-1}=\sum_{j=1}^{n-1} f\left(H_{j}(x)\right) S x_{n-j},
$$

and $S^{-1} T P x=\sum_{j=1}^{n-1} f\left(H_{j}(x)\right) x_{n-j}$, as $S_{1}: X_{1} \rightarrow Y_{1}$ is injective, as well as $\left(S^{-1} T\right)^{2} P x=\sum_{j=2}^{n-1} f\left(H_{j}(x)\right) x_{n-j+1}$. Step by step, we obtain $\left(S^{-1} T\right)^{n} P x$ $=0$.

We now prove that

$$
N(S) \subset X_{2}
$$

Let $x \in X=X_{1} \oplus X_{2}, x=x_{1}+x_{2}$ with $x_{1} \in X_{1}$ and $x_{2} \in X_{2}$. Assume that $S x=0$, i.e. $S x_{1}=-S x_{2}$. As $S x_{1} \in Y_{1}$ and $S x_{2} \in Y_{2}$, it follows that $S x_{1}=S x_{2}=0$. We thus have $x_{1} \in N(S) \cap X_{1}=\{0\}$, hence $x_{1}=0$.

Therefore we can consider $S^{-1} T: X_{2} \rightarrow X_{2}$. As $X=X_{2} \oplus X_{1}, N(T)=$ $N\left(T_{2}\right) \oplus \mathbb{C} x_{n-1}$.

Step 3. Now we finish the proof. We have to separate two cases. First, assume that (1) is true, i.e. $N(T) \subset_{\mathrm{e}} D(T: S)$. We have $N(T)=N\left(T_{2}\right) \oplus$ 
$\mathbb{C} x_{n-1}$ and $D(T: S)=D\left(T_{2}: S_{2}\right)$, as $\left.S^{-1} T\right|_{X_{1}}$ is nilpotent. So

$$
N(T) /[N(T) \cap D(T: S)] \cong N\left(T_{2}\right) /\left[N\left(T_{2}\right) \cap D\left(T_{2}: S_{2}\right)\right] \times \mathbb{C} x_{n-1} .
$$

Hence

$$
\operatorname{dim}\left(N\left(T_{2}\right) /\left[N\left(T_{2}\right) \cap D\left(T_{2}: S_{2}\right)\right]\right)=\operatorname{dim}(N(T) /[N(T) \cap D(T: S)])-1 .
$$

If $\operatorname{dim}(N(T) /[N(T) \cap D(T: S)])=1$, we stop. Otherwise, we reiterate the operation with $X$ replaced by $N(P)$ and $Y$ by $N(Q)$. We obtain two new projections $P_{1}$ and $Q_{1}$. Thus, in a finite number of steps, we will obtain

$$
\left\{\begin{array}{l}
X=\left(\bigcap_{j=0}^{k-1} N\left(P_{j}\right)\right) \oplus\left(\bigoplus_{j=0}^{k-1} R\left(P_{j}\right)\right), \\
Y=\left(\bigcap_{j=0}^{k-1} N\left(Q_{j}\right)\right) \oplus\left(\bigoplus_{j=0}^{k-1} R\left(Q_{j}\right)\right), \\
T_{2} \text { has the property } P\left(S_{2}: 0\right) .
\end{array}\right.
$$

Now, assume that (2) is true, i.e. $N(T: S) \subset_{\mathrm{e}} S^{-1} R(T)$. We have $N_{k}(T: S)=N_{k}\left(T_{1}: S_{1}\right) \oplus N_{k}\left(T_{2}: S_{2}\right)$ for each $k \in \mathbb{N}$. As $\left.S^{-1} T\right|_{X_{1}}$ is nilpotent of index $\leq n$, we get

$$
N_{k}\left(T_{1}: S_{1}\right)=X_{1} \quad \text { for every } k \geq n .
$$

In fact, $N_{k}\left(T_{1}: S_{1}\right)$ is clearly a subset of $X_{1}$. Conversely, let $x \in X_{1}$. We want to prove that $x \in N_{n}\left(T_{1}: S_{1}\right)$, i.e. we want to find $a_{0}, \ldots, a_{n}$ such that $a_{0}=$ $x, a_{n}=0$ and $T a_{i}=S a_{i+1}$. We have $x=P(x)=\sum_{j=0}^{n-1} f\left(H_{j}(x)\right) x_{n-j-1}$, so

$$
T x=\sum_{j=0}^{n-1} f\left(H_{j}(x)\right) T x_{n-j-1}=\sum_{j=1}^{n-1} f\left(H_{j}(x)\right) S x_{n-j} .
$$

Hence we can put $a_{1}=\sum_{j=1}^{n-1} f\left(H_{j}(x)\right) x_{n-j}$. Then

$$
T a_{1}=\sum_{j=2}^{n-1} f\left(H_{j}(x)\right) S x_{n-j+1},
$$

and we can put $a_{2}=\sum_{j=2}^{n-1} f\left(H_{j}(x)\right) x_{n-j+1}$. Step by step, we find the desired $a_{i}$, and we prove that $x \in N_{n}\left(T_{1}: S_{1}\right)$.

So $N(T: S)=X_{1} \oplus N\left(T_{2}: S_{2}\right)$. Moreover, $D_{1}(T: S)=D_{1}\left(T_{1}: S_{1}\right) \oplus$ $D_{1}\left(T_{2}: S_{2}\right)$. We have already shown that $D_{1}\left(T_{1}: S_{1}\right)=S^{-1} T P X=\left\langle x_{i}\right\rangle_{i=1}^{n-1}$. Thus

$$
\begin{aligned}
N(T: S) /\left[N(T: S) \cap D_{1}(T: S)\right] \cong & N\left(T_{2}: S_{2}\right) /\left[N\left(T_{2}: S_{2}\right) \cap D_{1}\left(T_{2}: S_{2}\right)\right] \\
& \times X_{1} /\left\langle x_{i}\right\rangle_{i=1}^{n-1} .
\end{aligned}
$$


As $X_{1} /\left\langle x_{i}\right\rangle_{i=1}^{n-1}=\mathbb{C} x_{0}$, we get

$$
\begin{aligned}
\operatorname{dim}\left(N\left(T_{2}: S_{2}\right) /\left[N \left(T_{2}:\right.\right.\right. & \left.\left.\left.S_{2}\right) \cap D_{1}\left(T_{2}: S_{2}\right)\right]\right) \\
& =\operatorname{dim}\left(N(T: S) /\left[N(T: S) \cap D_{1}(T: S)\right]\right)-1 .
\end{aligned}
$$

By Lemma 2.5, $T_{2}$ has the property $P\left(S_{2}: 0\right)$ if and only if $N\left(T_{2}: S_{2}\right) \subset$ $D_{1}\left(T_{2}: S_{2}\right)$. Thus, we can complete the proof in the same way as in the first case.

2.9. Remarks. (1) In the particular case where $X=Y$ and $S=I$, we recover the result on the essentially s-regular operators proved by V. Müller and V. Rakočević (see [8, Theorem 3.1] and [9]).

(2) Under condition (4) of Theorem 2.3, as $S_{1}^{-1} T_{1}$ is nilpotent and $S_{1}: X_{1} \rightarrow Y_{1}$ is bijective, we have $\sigma\left(T_{1}: S_{1}\right)=\left\{\lambda \in \mathbb{C} ; T_{1}-\lambda S_{1}\right.$ is not invertible $\}=\{0\}$.

Acknowledgements. I wish to thank my supervisor M. Mbekhta for discussions about the topic of this paper.

\section{References}

[1] C. Apostol, The reduced minimum modulus, Michigan Math. J. 32 (1985), 279-294.

[2] S. Grabiner, Uniform ascent and descent of bounded operators, J. Math. Soc. Japan 34 (1982), 317-337.

[3] M. A. Kaashoek, Stability theorems for closed linear operators, Nederl. Akad. Wetensch. Proc. Ser. A 68 = Indag. Math. 27 (1965), 452-466.

[4] T. Kato, Perturbation Theory for Linear Operators, Springer, Berlin, 1966.

[5] - Perturbation theory for nullity, deficiency and other quantities of linear operators, J. Anal. Math. 6 (1958), 261-322.

[6] M. Mbekhta, Résolvant généralisé et théorie spectrale, J. Operator Theory 21 (1989), 69-105.

[7] M. Mbekhta et A. Ouahab, Opérateurs s-réguliers dans un espace de Banach et théorie spectrale, Acta Sci. Math. (Szeged) 59 (1994), 525-543.

[8] V. Müller, On the regular spectrum, J. Operator Theory 31 (1994), 363-380.

[9] V. Rakočević, Generalized spectrum and commuting compact perturbations, Proc. Edinburgh Math. Soc. 36 (1993), 197-209.

Département de Mathématiques

Université de Lille I

U.M.R., C.N.R.S. 8524

59655 Villeneuve d'Ascq Cedex, France

E-mail: dominique.gagnage@agat.univ-lille1.fr 